\title{
IDENTIFICATION OF LOADS OCCURRING IN THE LUMBAR SPINE SECTION DURING THE ACTION OF LIFTING OBJECTS
}

\author{
K. Nowakowska*, R. Michnik ${ }^{* *}$, A. Myśliwiec ${ }^{* * *}$, H. Zadoń ${ }^{\dagger}$
}

\begin{abstract}
This work aimed to determine loads in the musculoskeletal system of the lumbar spine while lifting $10-\mathrm{kg}$ and 20-kg objects off a table. Tests of kinematics of the analyzed actions of lifting were conducted in a group of parents of disabled children. The parents suffered from chronic pain in the lower section of the spine. The action of lifting was divided into 3 positions: 1 - a moment of detachment of the object from the table, 2 - an intermediate stage, 3 - holding the object while adopting an upright posture. The identification of loads exerted on the musculoskeletal system was carried out using a mathematical model of the human body in the Anybody Modeling System. The solution of the inverse dynamics task using the techniques of static optimization was applied. It enabled the determination of the reaction values in the joints of the lumbar spine section as well as the estimation of the values of muscle forces. The obtained results were standardized according to the body weight (BW) of the examined person. The highest values of loads were obtained in segment L5-S1: $2.36 \pm 0.44 \mathrm{~N} / \mathrm{BW}$ for lifting an object of a mass of $10 \mathrm{~kg}$ as well as $3.09 \pm 0.41 \mathrm{~N} / \mathrm{BW}$ for lifting a 20-kg object. The increase of the object mass from $10 \mathrm{~kg}$ to $20 \mathrm{~kg}$ caused the increase of loads in all lumbar segments of the spine by around 31\% for position 1 and by $56 \%$ for position 3 . The most active muscles during the action of lifting were the back extensor muscle and transversus abdominis muscle.
\end{abstract}

Keywords: anybody modeling system, mathematical modeling, lifting, loads, lumbar spine

\section{Introduction}

Long-term overload of the motor organ usually results in its damage or pain ailments. Parents of disabled children are a group which is particularly vulnerable to the occurrence of pain disorders in the lower part of the back. Everyday caring and nursing activities are usually done by them in uncomfortable positions, which include long-term leaning of the torso, as the parents lift their children many times during a day (Nowakowska et al., 2017a). In recent years there have been numerous research works dealing with the application of a quantitative analysis of movement and biomechanical models of the human motor organ for different purposes, including the assessment of loads in the lumbar spine section (Damsgaard et al., 2006, Joszko et al., 2017, Jurkojć et al., 2017, Michnik et al., 2017, Nowakowska et al., 2017a, 2017b, Stambolian et al., 2016). The results show the consequences which may occur in the course of care of a child whose movement is impaired (Arjmand 2006, Nowakowska, 2017a). This work aimed to determine the loads in the musculoskeletal system of the lumbar spine section during the action of lifting objects. The researchers used the method of mathematical modelling and static optimization.

\footnotetext{
Katarzyna Nowakowska, MSc. Eng.: Department of Biomechatronics, Faculty of Biomedical Engeenering, Silesian Univesity of Technology; Poland,Katarzyna.Nowakowska@polsl.pl

** $\quad$ Robert Michnik, PhD. DSc. Eng: Department of Biomechatronics, Faculty of Biomedical Engeenering, Silesian Univesity of Technology; Poland, Robert.Michnik@polsl.pl

**** Andrzej Myśliwiec, PhD. DSc. Eng: Department of Kinesytherapy and Special Methods of Physiotherapy, Academy of Physical Education in Katowice; Poland, a.mysliwiec@awf.katowice.pl

† Hanna Zadoń, MSc. Eng.: Students Scientific „BIOKREATYWNI“, Faculty of Biomedical Engeenering, Silesian Univesity of Technology; Poland, hadonia@interia.pl
} 


\section{Methods}

A test group consisted of 30 women, mothers of children with motor disabilities (age: $43 \pm 9$ years old, body weight: $65.30 \pm 7.32 \mathrm{~kg}$, body height: $161.95 \pm 3.92 \mathrm{~cm}$ ), complaining of chronic pains in the lower spine section. The tests encompassed parents of disabled persons at the age of 6-25 who were qualified as level 4 or 5 according to the the Gross Motor Function Classification System (GMFCS). The criteria of qualification for the tests were as follows: a lack of visible posture defects and a lack of injuries over the past 3 months. The tests of kinematics of the movement of lifting the weight of $10 \mathrm{~kg}$ and $20 \mathrm{~kg}$ off the table were recorded by means of an optic system APAS. Displacements of markers during motion were recorded using one digital video camera SONY HDR-CX625, placed perpendicularly to the sagittal plane of motion. The frequency of sampling was $25 \mathrm{~Hz}$, which was deemed sufficient for the analyzed activities (they were not done very fast). No effect of blurring of markers was observed. The films were recorded in a full HD - high definition of the picture $(1920 \times 1080)$ in the.$m t s$ format. To determine the kinematics of the analyzed positions, six fluorescent markers were placed on the body of the examined person. These markers made it possible to determine a position of the axis of the rotation of joints. Markers were stuck on the: (1) wrist from the side of the small finger, (2) epicondylus of the humeral bone, (3) acromioclavicular joint, (4) trochanter major of the femoral bone, (5) lateral epicondylus of the femoral bone, (6) lateral ankle. The movement was recorded perpendicularly to the sagittal plane. For each recorded movement 3 positions were identified: 1 - a moment of detachment of the object from the table, the biggest leaning of the torso, 2 - an intermediate stage, 3 - holding the weight while adopting an upright posture. Position no 1 - the lifting of an object off the table - was identified on the basis of a recorded video film. The researchers indicated the first number of the film exposure in which no part of the object had any contact with the table. On the basis of the markers' positions, the angular values in joints were determined (in the APAS software programme). The values were entered directly into the Anybody system. In the model in the Anybody environment, the positions of markers were not defined (Fig. 1).
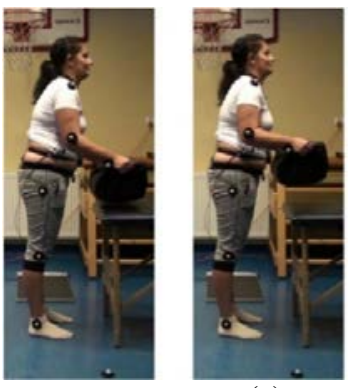

(a)

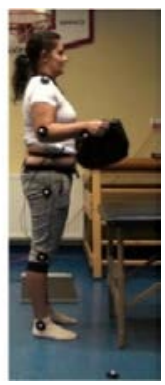

Fig. 1: Division of the movement of lifting the weight off the table: a) during experimental tests, $b$ ) in the Anybody Modeling System

The authors used a modified model of the whole body, the so-called 'Standing Model', which was accessible in the software library (Damsgaard et al., 2006). The applied model consisted of 69 rigid bodies (rigid solids) representing segments of the human body. These segments were connected by means of kinematic pairs. The pairs had a degree of freedom depending on the anatomical condition of the joints. The muscular system was modelled using over 1000 active force elements. The line of action of muscles depended on specific points of the initial and final attachment. The location of joints as well as points of muscle attachments were defined in local systems of coordinates of a given segment. The musculoskeletal model was scaled to the anatomical dimensions of a tested person. The model was adapted to the dimensions of the people tested using a method of linear scaling (different from default one). The method of 'ScalingLengthMassFatExt' took into consideration the tested person's height, mass and the percentage of fat tissue. A multibody model of the lumbar spine contained 7 rigid bodies, i.e. 5 lumbar vertebrae, the pelvis and a part of the thorax. It had 18 degrees of freedom. Intervertebral joints were modelled as balland-socket joints having 3 degrees of freedom. The model of the lumbar spine section included also 154 actuators representing muscles of the back and abdomen as well as a model of intra-abdominal pressure (de Zee et al., 2007). The lifting of the weight was simulated by applying vectors of force to the centre of each hand. The vector force amounted to $-49.1 \mathrm{~N}$ in the case of lifting a 10-kg object and $-98.1 \mathrm{~N}$ while lifting $20 \mathrm{~kg}$. During the development of the model the following simplifications were adopted: a) symmetricality of the human body was assumed; b) the movement was treated as flat symmetrical motion in the sagittal plane; c) the model's feet during the movement were in a constant contact with the ground; 
d) the weight which was being lifted was presented as vectors of force applied to the centre of the hands; e) due to the methodology of experimental tests the actual hunching of the back was not represented; f) the loads occurring in the spine were determined only for selected stages of each action. For each analyzed position, the location of the CoM was corrected by entering the value of its displacement in the sagittal plane. The displacement was determined on the basis of the mass and length of particular body segments obtained as a result of the process of scaling and on the basis of the Dempster coefficients. An application written in the MATLAB environment was used for this purpose. The identification of loads in the musculoskeletal system was conducted using the inverse dynamics task and applying static optimization.

The adopted optimization criterion was the criterion of motion control. It took into account the minimization of the sum of cubes of the proportion (ratio) of muscle forces to their maximum forces. As a limiting condition, it was assumed that resultant moments of external forces obtained from the inverse dynamics task were counterbalanced by the sum of moments of muscle forces having impact on a given joint. Moreover, an assumption was made that the muscle forces may adopt values in the range from zero to defined maximum values. The model was verified by means of the comparison with the results obtained from in-vivo tests and results from biomechanical model conducted by authors of other works (Arjmand, 2006, Rohlmann et al., 2014, Wilke et al., 1999) and measurements of the EMG activity recorded for the back extensor muscle (Nowakowska et al., 2017b). The values analyzed in this paper included resultant values of reactions in joints occurring at individual levels of the lumbar spine section. These values were standardized in relation to the weight of a tested person (BW - body weight) and the values of selected muscle forces.

\section{Results and discussion}

The highest loads during the analyzed action of lifting were recorded in the first phase of the movement, at the moment of detachment of the object from the table as well as during the biggest leaning of the torso. The loads were decreasing as the body was adopting an upright position. The highest load values were obtained in segment L5-S1. For the analyzed types of lifting they amounted to: $2.36 \pm 0.44 \mathrm{~N} / \mathrm{BW}$ for lifting a 10-kg weight and $3.09 \pm 0.41 \mathrm{~N} / \mathrm{BW}$ for lifting a 20-kg object (Fig. 1). The increase of the mass of the lifted weight from $10 \mathrm{~kg}$ to $20 \mathrm{~kg}$ caused the increase of loads in all segments of the lumbar spine section by around 31\% for position 1 and by 56\% for position 3 (holding the object while adopting an upright position). In addition to that, the comparison of the obtained values of the resultant reaction in joint L5-S1, between position 1 and 3, revealed that at an upright body posture loads diminished by $40 \%$ in the case of lifting a 10-kg object and by $29 \%$ in the case of a $20-\mathrm{kg}$ object. Figure 2 compiles load values obtained in segment L5-S1 during the action of lifting an object. They were standardized in relation to an upright position (100\%). In the course of lifting, the back extensor was the most active muscle of the back and its highest activity was recorded while lifting $20 \mathrm{~kg}:-1.49 \pm 0.22 \mathrm{~N} / \mathrm{BW}$. The maximum value of the muscle force while lifting $10 \mathrm{~kg}$ amounted to $1.12 \pm 0.24 \mathrm{~N} / \mathrm{BW}$. Among abdominal muscles, transverse abdominal muscles showed the highest activity. While lifting a 10-kg object, the maximum value of the muscle force equalled $0.82 \pm 0.12 \mathrm{~N} / \mathrm{BW}$, whereas in the case of lifting $20 \mathrm{~kg}$ it equalled: $1,09 \pm 0,15 \mathrm{~N} / \mathrm{BW}$. In the case of other abdominal muscles, no high values of muscle forces were recorded.

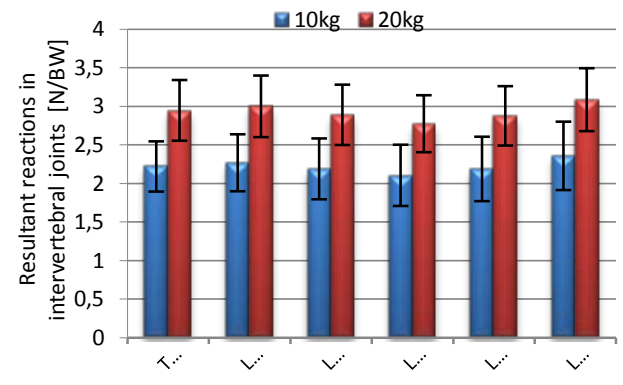

(a)

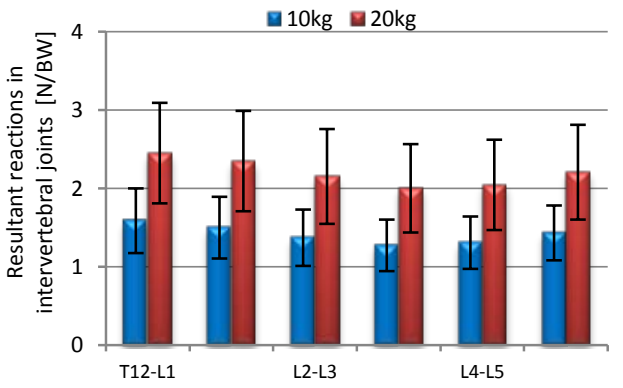

(b)

Fig. 2: Mean resultant values of reaction forces in the lumbar spine section while lifting 10-kg and 20-kg objects: a) position I, b) position III 


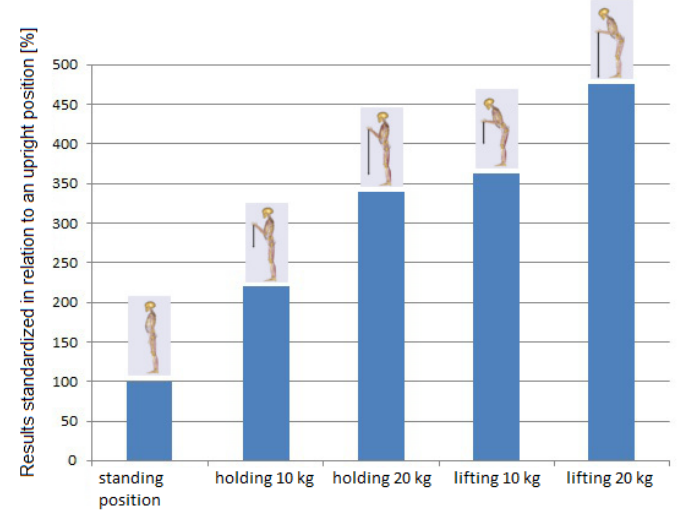

Fig. 3: Comparison of loads occurring in segment L5-S1 during the action of lifting.
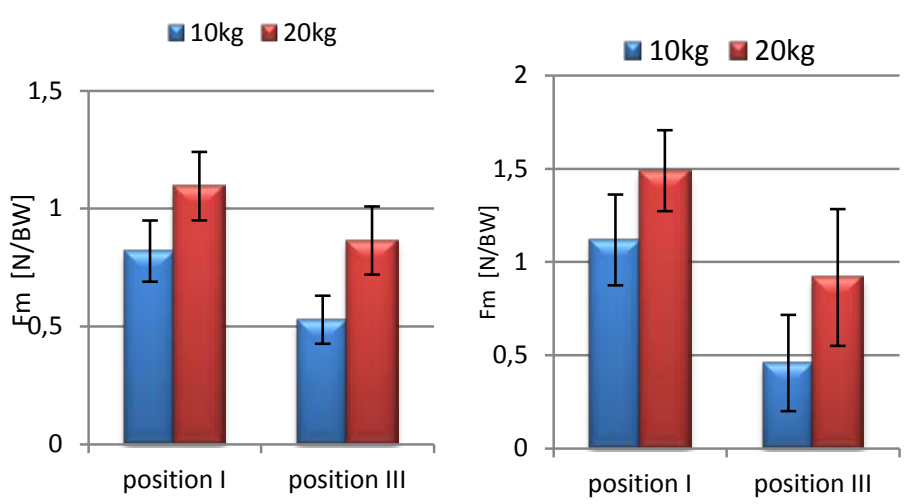

Fig. 4: Mean values of muscle forces during the action of lifting 10-kg and 20-kg objects: a) back extensor, b) transverse abdominal muscle.

\section{Conclusions}

The analysis of results obtained from the simulations conducted by means of a mathematical model of the human motor system revealed that the performance of simple everyday activities may lead to the occurrence of dangerously high loads in the lumbar spine section. The action of lifting a 10-kg weight may generate surprisingly high loads amounting to over $350 \%$ in relation to a standing position, whereas the action of lifting a $20-\mathrm{kg}$ object may reach as much as $475 \%$ in comparison with the loads occurring in a standing position. Such loads considerably decrease (even by over $30 \%$ ) when the weight is held in an upright position. The most active muscles during the action of lifting are: the back extensor muscle and the transverse abdominal muscle, whose force values decrease along with the reduction of the weight value and with the adoption of an upright position. The obtained values indicate that factors influencing the reduction of loads are as follows: avoiding the trunk's leaning (displacement of the centre of mass forward), lifting the object as close as possible to the body as well as proper tension of the muscles of the back and abdomen.

\section{References}

Arjmand N., Shirazi-Adl A. (2006) Model and in vivo studies on human trunk load partitioning and stability in isometric forward flexions. Journal of Biomechanics, 39, pp. 510-521

Damsgaard, M., Rasmussen, J., Christensen, S.T., Surma, E., de Zee, M. (2006) Analysis of musculoskeletal systems in the AnyBody Modeling System. Simul. Model. Pract. Theory, 14, pp. 1100-1111.

Joszko, K., Gzik, M., Wolański, W., Gzik-Zroska, B., Kawlewska, E. (2017) Biomechanical evaluation of human lumbar spine in spondylolisthesis. Journal of Applied Biomedicine, DOI 10.1016/j.jab.2017.10.004.

Jurkojć J., Wodarski P., Michnik R., Nowakowska K., Bieniek A., Gzik M. (2017) The Upper Limb Motion Deviation Index: A new comprehensive index of upper limb motion pathology. Acta of Bioengineering and Biomechanics, 19, 2, pp.175-185.

Michnik R., Nowakowska K., Jurkojć J., Jochymczyk-Woźniak K., Kopyta I. (2017) Motor functions assessment method based on energy changes in gait cycle. Acta of Bioengineering and Biomechanics, 19, 4, pp. 63-75.

Nowakowska K., Gzik M., Michnik R., Myśliwiec A., Jurkojć J., Suchoń S and, Burkacki M. (2017) The loads acting on lumbar spine during sitting down and standing up. Innovations in biomedical engineering, Gzik M., Tkacz E., Paszenda Z., Piętka E. (eds.) (in:) Advances in Intelligent Systems and Computing, 526, pp. 169-176.

Nowakowska K., Michnik R., Myśliwiec A., Chrzan M. (2017) Impact of strengthening of the erector spinae muscle on the values of loads of the muskuloskeletal system in the lumbar spine section. Engineering Mechanics 2017, Brno University of Technology, Brno, pp. 718-721.

Rohlmann A., Pohl D., Bender A., Graichen F. and Dymke J. (2014) Activities of Everyday Life with High Spinal Loads. PloS ONE, 9, 5, e98510.

Wilke, H.J., Neef, P., Caimi, M., Hoogland, T., Claes, L.E. (1999) New in vivo measurements of pressures in the intervertebral disc in daily life. Spine (Phila. Pa. 1976), 24, 755-62.

Zee de M., Hansen L., Wong Ch., Rasmussen J. and Simonsen E.B. (2007) A generic detailed rigid-body lumbar spine model. Journal of Biomechanics, 40, pp. 1219-1227. 